\title{
Hyperon bulk viscosity in the presence of antikaon condensate
}

\author{
Debarati Chatterjee ${ }^{1}$ and Debades Bandyopadhyay ${ }^{1}$ \\ Theory Division, Saha Institute of Nuclear Physics, 1/AF Bidhannagar, Kolkata-700064, \\ India
}

\begin{abstract}
We investigate the hyperon bulk viscosity due to the non-leptonic process $n+p \rightleftharpoons p+\Lambda$ in $K^{-}$condensed matter and its effect on the r-mode instability in neutron stars. We find that the hyperon bulk viscosity coefficient in the presence of antikaon condensate is suppressed compared with the case without the condensate. The suppressed hyperon bulk viscosity in the superconducting phase is still an efficient mechanism to damp the r-mode instability in neutron stars.
\end{abstract}

Subject headings: dense matter - stars:neutron - stars:oscillations - instabilities

\section{Introduction}

The study of r-modes in rotating neutron stars has generated great interest in recent times from two points of view. On one hand, r-modes of rotating neutron stars could be possible sources of detectable gravitational waves. On the other hand, the gravitational radiation driven instability of r-modes (Chandrasekhar 1970; Friedman 1978; Friedman \& Schutz 1978a.b; Friedman \& Morsink 1998; Lindblom et al. 1998; Andersson 1998; Andersson et al. 1999; Andersson \& Kokkotas 2001; Andersson 2003; Stergioulas 2003) might play an important role in regulating spins of young as well as old, accreting neutron stars in low mass $\mathrm{x}$-ray binaries(LMXBs). The latter situation has been strengthened by the fact that the spin distribution of pulsars has an upper limit at $730 \mathrm{~Hz}$ Chakrabarty 2005; Chakrabarty et al. 2003). It is worth mentioning here that the fastest rotating pulsar has a spin frequency 716 $\mathrm{Hz}$ (Hessels et al. 2006).

The bulk viscosity of neutron star matter is an important issue in connection with the damping of the r-mode instability in rotating neutron stars. The r-mode instability may be

\footnotetext{
${ }^{1}$ Centre for Astroparticle Physics, Saha Institute of Nuclear Physics, 1/AF Bidhannagar, Kolkata-700064, India
} 
suppressed through different mechanisms. It was shown that the damping of the instability by the large hyperon bulk viscosity coefficient could be an efficient mechanism (Jones 2001a.b; Lindblom \& Owen 2002; Haensel et al. 2002; Dalen \& Dieperink 2004; Drago et al. . 2005; Nayyar \& Owen 2006; Chatterjee \& Bandyopadhyay 2006, 2007a). However, it may be suppressed by several orders of magnitude when neutrons, protons or hyperons are superfluid (Haensel et al. 2000, 2001, 2002; Nayyar \& Owen 2006; Andersson 2007). This study was later extended to the calculation of bulk viscosity due to other exotic forms of matter such as unpaired quark matter (Madsen 1992, 2000; Dong et al. 2007a,b), quark-hadron mixed phase (Drago et al., 2005; Pan et al. 2006) and color superconducting quark matter (Alford \& Schmitt 2007a; Alford et al. 2007b, c; Sa'd et al. 2007a,b). Another possibility of damping the r-mode instability is the mutual friction between inter-penetrating neutron and proton superfluids (Lindblom \& Mendell 2000; Andersson 2007).

The superfluidity of neutron star matter plays a significant role on the damping of the r-modes in neutron stars. It was shown that superfluid particles taking part in non-leptonic weak processes involving hyperons might reduce the hyperon bulk viscosity coefficient by several orders of magnitude (Haensel et al. 2002; Andersson 2007). Recently, we have investigated the bulk viscosity due to the non-leptonic weak process $n \rightleftharpoons p+K^{-}$in $K^{-}$condensed matter (Chatteriee \& Bandyopadhyav 2007b). The kaon bulk viscosity was suppressed in the condensed phase by several orders of magnitude and could not damp the r-mode instability. This motivates us to further investigate how the bulk viscosity due to the non-leptonic process $n+p \rightleftharpoons p+\Lambda$ behaves in antikaon condensed matter and how it influences r-modes of neutron stars.

We organise the paper in the following way. In section 2, the field theoretical models of strong interactions, different phases of dense matter, the bulk viscosity coefficient and the corresponding time scale are described. Results of our calculation are discussed in section 3. Section 4 gives summary and conclusions.

\section{Formalism}

Our motivation is to calculate the hyperon bulk viscosity due to the non-leptonic process $n+p \rightleftharpoons p+\Lambda$ in a superconducting phase i.e. antikaon condensed phase. This process was extensively investigated in hadronic matter by several groups (Jones 2001a,b; Lindblom \& Owen 2002; Dalen \& Dieperink 2004; Navyar \& Owen 2006; Chatterjee \& Bandyopadhyay 2006, 2007a). In this case, we consider a first order phase transition from hadronic to $K^{-}$condensed matter. Both the pure hadronic and $K^{-}$condensed matter are described within the framework of relativistic field theoretical models. The constituents of matter in both phases 
are neutrons $(n)$, protons $(p), \Lambda$ hyperons, electrons and muons. The baryon-baryon interaction is mediated by the exchange of $\sigma, \omega$ and $\rho$ mesons and two strange mesons, scalar meson, $f_{0}(975)$ (denoted hereafter as $\sigma^{*}$ ) and the vector meson $\phi(1020)$ (Schaffner \& Mishustin 1996). Both phases maintain local charge neutrality and beta-equilibrium conditions whereas these conditions are satisfied globally in the mixed phase. Further baryons are embedded in the $K^{-}$condensate phase. We describe the equation of state (EoS) in the antikaon condensed phase using the following Lagrangian density for baryons (Schaffner \& Mishustin 1996)

$$
\begin{aligned}
\mathcal{L}_{B}= & \sum_{B} \bar{\Psi}_{B}\left(i \gamma_{\mu} \partial^{\mu}-m_{B}+g_{\sigma B} \sigma-g_{\omega B} \gamma_{\mu} \omega^{\mu}-g_{\rho B} \gamma_{\mu} \mathrm{t}_{B} \cdot \boldsymbol{\rho}^{\mu}\right) \Psi_{B} \\
& +\frac{1}{2}\left(\partial_{\mu} \sigma \partial^{\mu} \sigma-m_{\sigma}^{2} \sigma^{2}\right)-U(\sigma) \\
& -\frac{1}{4} \omega_{\mu \nu} \omega^{\mu \nu}+\frac{1}{2} m_{\omega}^{2} \omega_{\mu} \omega^{\mu}-\frac{1}{4} \boldsymbol{\rho}_{\mu \nu} \cdot \boldsymbol{\rho}^{\mu \nu}+\frac{1}{2} m_{\rho}^{2} \boldsymbol{\rho}_{\mu} \cdot \boldsymbol{\rho}^{\mu}+\mathcal{L}_{Y Y} .
\end{aligned}
$$

Here $\psi_{B}$ denotes the Dirac bispinor for baryons $B$ with vacuum mass $m_{B}$ and the isospin operator is $t_{B}$. The scalar self-interaction term (Boguta \& Bodmer 1977) is

$$
U(\sigma)=\frac{1}{3} g_{2} \sigma^{3}+\frac{1}{4} g_{3} \sigma^{4} .
$$

The Lagrangian density for hyperon-hyperon interaction $\left(\mathcal{L}_{Y Y}\right)$ is given by

$$
\begin{aligned}
\mathcal{L}_{Y Y}= & \sum_{B} \bar{\Psi}_{B}\left(g_{\sigma^{*} B} \sigma^{*}-g_{\phi B} \gamma_{\mu} \phi^{\mu}\right) \Psi_{B} \\
& +\frac{1}{2}\left(\partial_{\mu} \sigma^{*} \partial^{\mu} \sigma^{*}-m_{\sigma^{*}}^{2} \sigma^{* 2}\right)-\frac{1}{4} \phi_{\mu \nu} \phi^{\mu \nu}+\frac{1}{2} m_{\phi}^{2} \phi_{\mu} \phi^{\mu} .
\end{aligned}
$$

As nucleons do not couple with strange mesons, $g_{\sigma^{*} B}=g_{\phi B}=0$.

Similarly we treat the (anti)kaon-baryon interaction in the same footing as the baryonbaryon interaction. The Lagrangian density for (anti)kaons in the minimal coupling scheme is (Glendenning \& Schaffner-Bielich 1998, 1999; Banik \& Bandyopadhyay 2001a, b),

$$
\mathcal{L}_{K}=D_{\mu}^{*} \bar{K} D^{\mu} K-m_{K}^{* 2} \bar{K} K
$$

where the covariant derivative is $D_{\mu}=\partial_{\mu}+i g_{\omega K} \omega_{\mu}+i g_{\phi K} \phi_{\mu}+i g_{\rho K} \mathrm{t}_{K} \cdot \boldsymbol{\rho}_{\mu}$ and the effective mass of (anti)kaons is $m_{K}^{*}=m_{K}-g_{\sigma K} \sigma-g_{\sigma^{*} K} \sigma^{*}$.

We perform this calculation in the mean field approximation (Serot \& Walecka 1986). The mean meson fields in the condensed phase are denoted by $\sigma, \sigma^{*}, \omega_{0}, \phi_{0}$ and $\rho_{03}$. The expressions for mean fields can be found in Ref. (Banik \& Bandyopadhyay 2001b; Chatterjee \& Bandyopadhyay 2007b). The in-medium energy of $K^{-}$mesons for $s$-wave $(\vec{k}=0)$ condensation is given by

$$
\omega_{K^{-}}=\mu_{K^{-}}=m_{K}^{*}-g_{\omega K} \omega_{0}-g_{\phi K} \phi_{0}+I_{3 K^{-}} g_{\rho K} \rho_{03},
$$


where $\mu_{K^{-}}$is the chemical potential of $K^{-}$mesons and the isospin projection is $I_{3 K^{-}}=-1 / 2$. The chemical potential for baryons $B$ is given by

$$
\mu_{B}^{K}=\left(k_{F_{B}}^{2}+m_{B}^{* K^{2}}\right)^{1 / 2}+g_{\omega B} \omega_{0}+g_{\phi B} \phi_{0}+I_{3 B} g_{\rho B} \rho_{03}
$$

where effective baryon mass is $m_{B}^{* K}=m_{B}-g_{\sigma B} \sigma-g_{\sigma^{*} B} \sigma^{*}$ and isospin projection for baryons $B$ is $I_{3 B}$.

We obtain the mean fields in the hadronic phase putting source terms for $K^{-}$mesons equal to zero in corresponding equations of motion (Banik \& Bandyopadhyay 2001b; Chatterjee \& Bandyop 2007b).

The total energy density and pressure in the antikaon condensed phase are given by

$$
\begin{aligned}
\varepsilon^{K}= & \frac{1}{2} m_{\sigma}^{2} \sigma^{2}+\frac{1}{3} g_{2} \sigma^{3}+\frac{1}{4} g_{3} \sigma^{4}+\frac{1}{2} m_{\sigma^{*}}^{2} \sigma^{* 2}+\frac{1}{2} m_{\omega}^{2} \omega_{0}^{2}+\frac{1}{2} m_{\phi}^{2} \phi_{0}^{2}+\frac{1}{2} m_{\rho}^{2} \rho_{03}^{2} \\
& +\sum_{B=n, p, \Lambda} \frac{2 J_{B}+1}{2 \pi^{2}} \int_{0}^{k_{F_{B}}}\left(k^{2}+m_{B}^{* K^{2}}\right)^{1 / 2} k^{2} d k+\sum_{l=e^{-}, \mu^{-}} \frac{1}{\pi^{2}} \int_{0}^{K_{F_{l}}}\left(k^{2}+m_{l}^{2}\right)^{1 / 2} k^{2} d k \\
& +m_{K}^{*} n_{K^{-}},
\end{aligned}
$$

and

$$
\begin{aligned}
P^{K}= & -\frac{1}{2} m_{\sigma}^{2} \sigma^{2}-\frac{1}{3} g_{2} \sigma^{3}-\frac{1}{4} g_{3} \sigma^{4}-\frac{1}{2} m_{\sigma^{*}}^{2} \sigma^{* 2}+\frac{1}{2} m_{\omega}^{2} \omega_{0}^{2}+\frac{1}{2} m_{\phi}^{2} \phi_{0}^{2}+\frac{1}{2} m_{\rho}^{2} \rho_{03}^{2} \\
& +\frac{1}{3} \sum_{B=n, p, \Lambda} \frac{2 J_{B}+1}{2 \pi^{2}} \int_{0}^{k_{F_{B}}} \frac{k^{4} d k}{\left(k^{2}+m_{B}^{* K^{2}}\right)^{1 / 2}}+\frac{1}{3} \sum_{l=e^{-}, \mu^{-}} \frac{1}{\pi^{2}} \int_{0}^{K_{F_{l}}} \frac{k^{4} d k}{\left(k^{2}+m_{l}^{2}\right)^{1 / 2}}
\end{aligned}
$$

We describe the mixed phase of hadronic and $K^{-}$condensed matter using the Gibbs conditions for thermodynamic equilibrium along with global charge and baryon number conservation laws (Glendenning 1992; Glendenning \& Schaffner-Bielich 1999).

Now we focus on the calculation of bulk viscosity due to the non-leptonic process. It was shown that the real part of bulk viscosity coefficient was related to relaxation times of microscopic processes (Landau \& Liftshitz 1999; Lindblom \& Owen 2002) by the relation,

$$
\zeta=\frac{P\left(\gamma_{\infty}-\gamma_{0}\right) \tau}{1+(\omega \tau)^{2}},
$$

where $P$ is the pressure, $\tau$ is the net microscopic relaxation time and $\gamma_{\infty}$ and $\gamma_{0}$ are 'infinite' and 'zero' frequency adiabatic indices respectively and their difference

$$
\gamma_{\infty}-\gamma_{0}=-\frac{n_{b}^{2}}{P} \frac{\partial P}{\partial n_{n}} \frac{d \bar{x}_{n}}{d n_{b}}
$$


is determined using EoS as input. Here $\bar{x}_{n}=\frac{n_{n}}{n_{b}}$ gives the neutron fraction in the equilibrium state and $n_{b}=\sum_{B} n_{B}$ is the total baryon density. We are interested in $(1, \mathrm{~m}) \mathrm{r}$-mode. In this case, the angular velocity $(\omega)$ of the mode is related to the angular velocity $(\Omega)$ of a rotating neutron star as $\omega=\frac{2 m}{l(l+1)} \Omega$ (Andersson 2003).

The partial derivatives of pressure with respect to neutron fraction in both phases are calculated using the Gibbs-Duhem relation (Chatterjee \& Bandyopadhyay 2007b). In the pure hadronic phase, this relation gives

$$
\frac{\partial P^{h}}{\partial n_{n}^{h}}=n_{n}^{h} \alpha_{n n}^{h}+n_{p}^{h} \alpha_{p n}^{h}+n_{\Lambda}^{h} \alpha_{\Lambda n}^{h},
$$

where $\alpha_{i j}$ is defined by $\alpha_{i j}=\left(\frac{\partial \mu_{i}}{\partial n_{j}}\right)_{n_{k}, k \neq j}$. Similarly, in the pure condensed phase, it is given by

$$
\frac{\partial P^{K}}{\partial n_{n}^{K}}=n_{n}^{K} \alpha_{n n}^{K}+n_{p}^{K} \alpha_{p n}^{K}+n_{\Lambda}^{K} \alpha_{\Lambda n}^{K}+n_{K^{-}} \alpha_{K^{-} n}^{K} .
$$

In the mixed phase, this relation has the form

$$
\frac{\partial P}{\partial n_{n}}=\frac{\partial P^{h}}{\partial n_{n}^{h}}+\frac{\partial P^{K}}{\partial n_{n}^{K}}
$$

where $n_{n}=(1-\chi) n_{n}^{h}+\chi n_{n}^{K}$.

Next we calculate relaxation times of the non-leptonic process in different phases. In this case, we express all perturbed quantities in terms of the variation in neutron number density $\left(n_{n}\right)$ in the respective phase. The relaxation time $(\tau)$ for the non-leptonic process is given by (Lindblom \& Owen 2002)

$$
\frac{1}{\tau}=\frac{\Gamma_{\Lambda}}{\delta \mu} \frac{\delta \mu}{\delta n_{n}^{j}}
$$

Here, $\delta n_{n}^{j}=n_{n}^{j}-\bar{n}_{n}^{j}$ is the departure of neutron fraction from its thermodynamic equilibrium value $\bar{n}_{n}^{j}$ in the $\mathrm{j}$-th (=hadron, $K^{-}$condensed) phase. The reaction rate per unit volume for the non-leptonic process in question was already calculated by others (Lindblom \& Owen 2002; Navyar \& Owen 2006). The relaxation time $(\tau)$ for the process in the hadronic phase is given by (Lindblom \& Owen 2002)

$$
\frac{1}{\tau}=\frac{(k T)^{2}}{192 \pi^{3}} p_{\Lambda}<\left|M_{\Lambda}\right|^{2}>\frac{\delta \mu}{\delta n_{n}^{h}},
$$

along with

$$
\frac{\delta \mu}{\delta n_{n}^{h}}=\left(\alpha_{n n}^{h}-\alpha_{\Lambda n}^{h}\right)+\left(\alpha_{n p}^{h}-\alpha_{\Lambda p}^{h}\right) \frac{\delta n_{p}^{h}}{\delta n_{n}^{h}}+\left(\alpha_{n \Lambda}^{h}-\alpha_{\Lambda \Lambda}^{h}\right) \frac{\delta n_{\Lambda}^{h}}{\delta n_{n}^{h}},
$$


where $p_{\Lambda}$ is the Fermi momentum for $\Lambda$ hyperons and $<\left|M_{\Lambda}\right|^{2}>$ is the angle averaged matrix element squared in the hadronic phase given by Ref.(Lindblom \& Owen 2002; Nayyar \& Owen 2006). In pure hadronic phase, the second term in Eq.(16) vanishes because $\delta n_{p}^{h}=0$. However, the calculation of the hadronic part of the mixed phase is a little bit involved and described below.

The relaxation time in the antikaon condensed phase has the same form as in Eq.(15). In this case, the angle averaged matrix element , $\left\langle\left|M_{\Lambda}\right|^{2}\right\rangle$ and $p_{\Lambda}$ are to be calculated in the condensed phase. Also, we calculate $\frac{\delta \mu}{\delta n_{n}^{K}}$ from the chemical potential imbalance due to the non-leptonic hyperon process $n+p \rightleftharpoons p+\Lambda$ and it is given by,

$$
\begin{aligned}
\delta \mu & =\delta \mu_{n}^{K}-\delta \mu_{\Lambda}^{K} \\
& =\left(\alpha_{n n}^{K} \delta n_{n}^{K}+\alpha_{n p}^{K} \delta n_{p}^{K}+\alpha_{n \Lambda}^{K} \delta n_{\Lambda}^{K}+\alpha_{n K^{-}} \delta n_{K^{-}}\right) \\
& -\left(\alpha_{\Lambda n}^{K} \delta n_{n}^{K}+\alpha_{\Lambda p}^{K} \delta n_{p}^{K}+\alpha_{\Lambda \Lambda}^{K} \delta n_{\Lambda}^{K}+\alpha_{n K^{-}} \delta n_{K^{-}}\right) .
\end{aligned}
$$

We express $\delta \mu$ in terms of $\delta n_{n}^{K}$ and obtain $\frac{\delta \mu}{\delta n_{n}^{K}}$ using the following constraints,

$$
\begin{aligned}
\left(\delta n_{n}^{K}+\delta n_{p}^{K}+\delta n_{\Lambda}^{K}\right) & =0 \\
\left(\delta n_{p}^{K}-\delta n_{K^{-}}\right) & =0
\end{aligned}
$$

and the chemical equilibrium in the strangeness changing process $n \rightleftharpoons p+K^{-}$,

$$
\begin{aligned}
\delta \mu_{n}^{K}-\delta \mu_{p}^{K}-\delta \mu_{K^{-}} & =\left(\alpha_{n n}^{K} \delta n_{n}^{K}+\alpha_{n p}^{K} \delta n_{p}^{K}++\alpha_{n \Lambda}^{K} \delta n_{\Lambda}^{K}+\alpha_{n K^{-}} \delta n_{K^{-}}\right) \\
- & \left(\alpha_{p n}^{K} \delta n_{n}^{K}+\alpha_{p p}^{K} \delta n_{p}^{K}++\alpha_{p \Lambda}^{K} \delta n_{\Lambda}^{K}+\alpha_{p K^{-}} \delta n_{K^{-}}\right) \\
- & \left(\alpha_{K^{-}{ }_{n}} \delta n_{n}^{K}+\alpha_{K^{-} p} \delta n_{p}^{K}++\alpha_{K^{-} \Lambda} \delta n_{\Lambda}^{K}+\alpha_{K^{-} K^{-}} \delta n_{K^{-}}\right)=0
\end{aligned}
$$

Next we calculate $\alpha_{i j}$ in the hadronic as well as $K^{-}$condensed phases using the EoS. We can write down these quantities for both phases in generalised forms. For $B=B^{\prime}$, we get

$$
\begin{aligned}
\alpha_{B B^{\prime}}^{P} & =\frac{\partial \mu_{B}^{P}}{\partial n_{B^{\prime}}^{P}} \\
& =\left(\frac{g_{\omega B}}{m_{\omega}}\right)^{2}+\frac{1}{4}\left(\frac{g_{\rho B}}{m_{\rho}}\right)^{2}+\left(\frac{g_{\phi B}}{m_{\phi}}\right)^{2}+\frac{\pi^{2}}{k_{F_{B}} \sqrt{k_{F_{B}}^{2}+m_{B}^{* P^{2}}}} \\
& -\frac{m_{B}^{* P}}{\sqrt{k_{F_{B}}^{2}+m_{B}^{* P^{2}}}}\left(g_{\sigma B} \frac{\partial \sigma}{\partial n_{B^{\prime}}^{P}}+g_{\sigma^{*} B} \frac{\partial \sigma^{*}}{\partial n_{B^{\prime}}^{P}}\right),
\end{aligned}
$$

and for $B \neq B^{\prime}$

$$
\alpha_{B B^{\prime}}^{P}=\frac{\partial \mu_{B}^{P}}{\partial n_{B^{\prime}}^{P}}
$$




$$
\begin{aligned}
& =\left(\frac{g_{\omega B} g_{\omega B^{\prime}}}{m_{\omega}^{2}}\right)-\frac{1}{4}\left(\frac{g_{\rho B} g_{\rho B^{\prime}}}{m_{\rho}^{2}}\right) \\
& -\frac{m_{B}^{* P}}{\sqrt{k_{F_{B}}^{2}+m_{B}^{* P^{2}}}}\left(g_{\sigma B} \frac{\partial \sigma}{\partial n_{B^{\prime}}^{P}}+g_{\sigma^{*} B} \frac{\partial \sigma^{*}}{\partial n_{B^{\prime}}^{P}}\right),
\end{aligned}
$$

along with the following relations applicable for both cases

$$
\begin{gathered}
\frac{\partial \sigma}{\partial n_{B^{\prime}}^{P}}=\frac{\left(\frac{g_{\sigma B}}{m_{\sigma}^{2}}\right) \frac{m_{B}^{* P}}{\sqrt{k_{F_{B}}^{2}+m_{B}^{* P^{2}}}}}{D-D^{\prime} \times D^{\prime \prime}}, \\
\frac{\partial \sigma^{*}}{\partial n_{B^{\prime}}^{P}}=-D^{\prime \prime} \frac{\partial \sigma}{\partial n_{B^{\prime}}^{P}},
\end{gathered}
$$

where

$$
\begin{gathered}
D=1+\frac{1}{m_{\sigma}^{2}} \frac{d^{2} U}{d \sigma^{2}}+\sum_{B=n, p, \Lambda} \frac{\left(2 J_{B}+1\right)}{2 \pi^{2}}\left(\frac{g_{\sigma B}}{m_{\sigma}}\right)^{2} \int_{0}^{K_{F_{B}}} \frac{k^{4} d k}{\left(k^{2}+m_{B}^{* P^{2}}\right)^{3 / 2}}, \\
D^{\prime}=\sum_{B=n, p, \Lambda} \frac{\left(2 J_{B}+1\right)}{2 \pi^{2}}\left(\frac{g_{\sigma B} g_{\sigma^{*} B}}{m_{\sigma}^{2}}\right) \int_{0}^{K_{F_{B}}} \frac{k^{4} d k}{\left(k^{2}+m_{B}^{* P^{2}}\right)^{3 / 2}}, \\
D^{\prime \prime}=\frac{\sum_{B=n, p, \Lambda} \frac{\left(2 J_{B}+1\right)}{2 \pi^{2}}\left(\frac{g_{\sigma B} g_{\sigma^{*} B}}{m_{\sigma}^{* 2}}\right) \int_{0}^{K_{F_{B}}} \frac{k^{4} d k}{\left(k^{2}+m_{B}^{* P^{2}}\right)^{3 / 2}}}{1+\sum_{B=n, p, \Lambda} \frac{\left(2 J_{B}+1\right)}{2 \pi^{2}}\left(\frac{g_{\sigma^{*} B}}{m_{\sigma}^{*}}\right)^{2} \int_{0}^{K_{F_{B}}} \frac{k^{4} d k}{\left(k^{2}+m_{B}^{* P^{2}}\right)^{3 / 2}}} .
\end{gathered}
$$

Here $B$ and $B^{\prime}$ denote baryons and $\mathrm{P}$ stands for hadron $(h)$ or antikaon $(K)$ phase. Further nucleons do not couple with strange strange mesons i.e. $g_{\sigma^{*} N}=g_{\phi N}=0$. Similarly, $\Lambda$ hyperons do not couple with $\rho$ meson i.e. $g_{\rho \Lambda}=0$. The results for other $\alpha$ s in the antikaon condensed phase are given below,

$$
\begin{gathered}
\alpha_{B K^{-}}=\frac{\partial \mu_{B}^{K}}{\partial n_{K^{-}}} \\
=-\left(\frac{g_{\omega B} g_{\omega K}}{m_{\omega}^{2}}\right) \pm \frac{1}{4}\left(\frac{g_{\rho B} g_{\rho K}}{m_{\rho}^{2}}\right)-\left(\frac{g_{\phi B} g_{\phi K}}{m_{\phi}^{2}}\right) \\
-\frac{m_{B}^{* K}}{\sqrt{k_{F_{B}}^{2}+m_{B}^{* K^{2}}}}\left(g_{\sigma B} \frac{\partial \sigma}{\partial n_{K^{-}}}+g_{\sigma^{*} B} \frac{\partial \sigma^{*}}{\partial n_{K^{-}}}\right) \\
\alpha_{K^{-} B}=\frac{\partial \mu_{K^{-}}}{\partial n_{B}^{K}} \\
=-\left(\frac{g_{\omega B} g_{\omega K}}{m_{\omega}^{2}}\right) \pm \frac{1}{4}\left(\frac{g_{\rho B} g_{\rho K}}{m_{\rho}^{2}}\right)-\left(\frac{g_{\phi B} g_{\phi K}}{m_{\phi}^{2}}\right)-\left(g_{\sigma K} \frac{\partial \sigma}{\partial n_{B}^{K}}+g_{\sigma^{*} K} \frac{\partial \sigma^{*}}{\partial n_{B}^{K}}\right)
\end{gathered}
$$




$$
\alpha_{K^{-} K^{-}}=\frac{\partial \mu_{K^{-}}}{\partial n_{K^{-}}}=\left(\frac{g_{\omega K}}{m_{\omega}}\right)^{2}+\left(\frac{g_{\phi K}}{m_{\phi}}\right)^{2}+\frac{1}{4}\left(\frac{g_{\rho K}}{m_{\rho}}\right)^{2}-g_{\sigma K} \frac{\partial \sigma}{\partial n_{K^{-}}}-g_{\sigma^{*} K} \frac{\partial \sigma^{*}}{\partial n_{K^{-}}},
$$

where,

$$
\begin{aligned}
\frac{\partial \sigma}{\partial n_{K^{-}}} & =\frac{\frac{g_{\sigma K}}{m_{\sigma}^{2}}-\frac{g_{\sigma^{*} K} D^{\prime \prime}}{m_{\sigma}^{2}}}{D-D^{\prime} \times D^{\prime \prime}} \\
\frac{\partial \sigma^{*}}{\partial n_{K^{-}}} & =\frac{\frac{g_{\sigma^{*} K}}{m_{\sigma^{*}}^{2}}}{F}-D^{\prime \prime} \frac{\partial \sigma}{\partial n_{K^{-}}}
\end{aligned}
$$

and

$$
F=1+\sum_{B=n, p, \Lambda} \frac{\left(2 J_{B}+1\right)}{2 \pi^{2}}\left(\frac{g_{\sigma^{*} B}}{m_{\sigma}^{*}}\right)^{2} \int_{0}^{K_{F_{B}}} \frac{k^{4} d k}{\left(k^{2}+m_{B}^{* P^{2}}\right)^{3 / 2}} .
$$

In the second term in Eq.(27) and (28), + ve sign corresponds to neutrons and -ve is for protons. With the given $\alpha_{i j}$, we can now calculate relaxation time for the non-leptonic process in hadron as well as antikaon condensed phases. As soon as we know the relaxation time, we can calculate the bulk viscosity coefficient in each phase.

Now we focus on the calculation of relaxation time and bulk viscosity in the mixed phase. For this, we have to express the chemical imbalance $(\delta \mu)$ in the non-leptonic hyperon process as given by Eq.(17) in terms of $\delta n_{n}^{K}$ from the following constraints,

$$
\begin{aligned}
(1-\chi)\left(\delta n_{n}^{h}+\delta n_{p}^{h}+\delta n_{\Lambda}^{h}\right)+\chi\left(\delta n_{n}^{K}+\delta n_{p}^{K}+\delta n_{\Lambda}^{K}\right) & =0, \\
(1-\chi) \delta n_{p}^{h}+\chi\left(\delta n_{p}^{K}-\delta n_{K^{-}}\right) & =0, \\
\delta \mu_{p}^{h} & =\delta \mu_{p}^{K} \\
\delta \mu_{n}^{h} & =\delta \mu_{n}^{K}, \\
\delta \mu_{\Lambda}^{h} & =\delta \mu_{\Lambda}^{K} \\
\delta \mu_{n}^{K}-\delta \mu_{p}^{K}-\delta \mu_{K^{-}} & =0 .
\end{aligned}
$$

Here we have $\delta \chi=0$ because number densities deviate from their equilibrium values only by internal reactions (Lindblom \& Owen 2002). First two constraints follow from the conservation of baryon number and electric charge neutrality. The last constrain is the result of the chemical equilibrium involving $K^{-}$condensate as already shown by Eq.(19). The other constraints are due to the equality of neutron, proton and $\Lambda$ chemical potentials in the hadronic and condensed phases and we can rewrite them as

$$
\begin{aligned}
\left(\alpha_{p n}^{h} \delta n_{n}^{h}+\alpha_{p p}^{h} \delta n_{p}^{h}+\alpha_{p \Lambda}^{h} \delta n_{\Lambda}^{h}\right)-\left(\alpha_{p n}^{K} \delta n_{n}^{K}+\alpha_{p p}^{K} \delta n_{p}^{K}+\alpha_{p \Lambda}^{K} \delta n_{\Lambda}^{K}+\alpha_{p K^{-}} \delta n_{K^{-}}\right) & =0 \\
\left(\alpha_{n n}^{h} \delta n_{n}^{h}+\alpha_{n p}^{h} \delta n_{p}^{h}+\alpha_{n \Lambda}^{h} \delta n_{\Lambda}^{h}\right)-\left(\alpha_{n n}^{K} \delta n_{n}^{K}+\alpha_{n p}^{K} \delta n_{p}^{K}+\alpha_{n \Lambda}^{K} \delta n_{\Lambda}^{K}+\alpha_{n K^{-}} \delta n_{K^{-}}\right) & =0 \\
\left(\alpha_{\Lambda n}^{h} \delta n_{n}^{h}+\alpha_{\Lambda p}^{h} \delta n_{p}^{h}+\alpha_{\Lambda \Lambda}^{h} \delta n_{\Lambda}^{h}\right)-\left(\alpha_{\Lambda n}^{K} \delta n_{n}^{K}+\alpha_{\Lambda p}^{K} \delta n_{p}^{K}+\alpha_{\Lambda \Lambda}^{K} \delta n_{\Lambda}^{K}+\alpha_{\Lambda K^{-}} \delta n_{K^{-}}\right) & =0(33)
\end{aligned}
$$


We express $\delta n_{n}^{h}, \delta n_{p}^{h}, \delta n_{\Lambda}^{h}, \delta n_{p}^{K}, \delta n_{\Lambda}^{K}$ and $\delta n_{K^{-}}$in terms of $\delta n_{n}^{K}$ using above six constraints. For this purpose, we solve a $6 \times 6$ matrix constructed out of above six relations and obtain $\frac{\delta \mu}{\delta n_{n}^{K}}$. Similarly, we obtain $\frac{\delta \mu}{\delta n_{n}^{h}}$ in the mixed phase from the above constraints. This completes the calculation of relaxation time and bulk viscosity in the mixed phase.

Next we calculate critical angular velocity as a function temperature and mass of a rotating neutron star. The bulk viscosity damping timescale $\left(\tau_{B}\right)$ due to the non-leptonic process involving $\Lambda$ hyperons and the bulk viscosity profile as a function of $r$ are obtained following the Ref. (Lindblom et al. 1999; Lindblom \& Owen 2002; Nayyar \& Owen 2006; Chatterjee \& Bandyopadhyay 2006). Further we take into account time scales associated with gravitational radiation $\left(\tau_{G R}\right)$ (Lindblom et al. 1998), bulk viscosity due to modified Urca process $\left(\tau_{U}\right)$ involving only nucleons (Sawver 1989; Andersson \& Kokkotas 2001) and the shear viscosity $\left(\tau_{S V}\right)$ (Lindblom et al. 1998; Andersson \& Kokkotas 2001; Andersson 2007) and define the overall r-mode time scale $\left(\tau_{r}\right)$ as

$$
\frac{1}{\tau_{r}}=-\frac{1}{\tau_{G R}}+\frac{1}{\tau_{B}}+\frac{1}{\tau_{U}}+\frac{1}{\tau_{S V}}
$$

Finally, solving $\frac{1}{\tau_{r}}=0$, we calculate the critical angular velocity above which the r-mode is unstable whereas it is stable below the critical angular velocity.

\section{Results and Discussion}

For this calculation, nucleon-meson coupling constants are taken from Ref. Glendenning \& Moszkowski 1991) and this set is known as GM. Nucleon-meson coupling constants are determined by reproducing nuclear matter saturation properties such as binding energy $E / B=-16.3 \mathrm{MeV}$, baryon density $n_{0}=0.153 \mathrm{fm}^{-3}$, asymmetry energy coefficient $a_{\text {asy }}=32.5 \mathrm{MeV}$, incompressibility $K=300 \mathrm{MeV}$ and effective nucleon mass $m_{N}^{*} / m_{N}=0.70$. Further we need to know kaon-meson coupling constants and determine them using the quark model and isospin counting rule. The vector coupling constants are given by

$$
g_{\omega K}=\frac{1}{3} g_{\omega N} \quad \text { and } \quad g_{\rho K}=g_{\rho N} .
$$

The scalar coupling constant is obtained from the real part of $K^{-}$optical potential depth at normal nuclear matter density

$$
U_{\bar{K}}\left(n_{0}\right)=-g_{\sigma K} \sigma-g_{\omega K} \omega_{0}
$$

Antikaons experience an attractive potential whereas kaons has a repulsive interaction in nuclear matter (Friedman et al. 1994; Koch 1994; Batty et al. 1997; Waas \& Weise 1997; 
Li et al. 1997a,b; Friedman et al. 1999; Pal et al. 2000). The analysis of $K^{-}$atomic data using a hybrid model (Friedman et al. 1999) which combines the relativistic mean field approach in the nuclear interior and a phenomenological potential at low density, yielded the real part of the antikaon potential as large as $U_{\bar{K}}\left(n_{0}\right)=-180 \pm 20 \mathrm{MeV}$ at normal nuclear matter density. It was predicted that $K^{-}$condensation might occur in neutron star matter for strongly attractive antikaon potential $\sim-100 \mathrm{MeV}$ or more. In this calculation, we adopt the value of antikaon optical potential depth at normal nuclear matter density as $U_{\bar{K}}\left(n_{0}\right)=-160 \mathrm{MeV}$. We obtain kaon-scalar meson coupling constant $g_{\sigma K}=2.9937$ corresponding to this antikaon optical potential depth.

On the other hand, hyperon-vector meson coupling constants are determined using SU(6) symmetry of the quark model (Dover \& Gal 1984; Schaffner et al. 1994; Schaffner \& Mishustin 1996) and the scalar $\sigma$ meson coupling to $\Lambda$ hyperons is calculated from the hyperon potential depth in normal nuclear matter $U_{\Lambda}^{N}\left(n_{0}\right)=-30 \mathrm{MeV}$ obtained from hypernuclei data (Dover \& Gal 1984; Chrien \& Dover 1989). The hyperon- $\sigma^{*}$ coupling constant is determined from double $\Lambda$ hypernuclei data (Schaffner et al. 1993; Schaffner \& Mishustin 1996).

The strange meson fields also couple with (anti)kaons. The $\sigma^{*}$-K coupling constant determined from the decay of $f_{0}(925)$ is $g_{\sigma^{*} K}=2.65$ and the vector $\phi$ meson coupling with (anti)kaons $\sqrt{2} g_{\phi K}=6.04$ follows from the SU(3) relation (Schaffner \& Mishustin 1996).

The onsets of $K^{-}$condensate and $\Lambda$ hyperons in neutron star matter are sensitive to the composition of matter and the strength of antikaon optical potential depth (Banik \& Bandyopadhyay $2001 \mathrm{~b})$. It was further noted that the early appearance of either $\Lambda$ hyperons or the $K^{-}$ condensate delayed the onset of the other to higher densities. In this calculation, for $U_{\bar{K}}\left(n_{0}\right)=-160 \mathrm{MeV}, \Lambda$ hyperons appear just after the onset of $K^{-}$condensation. The $K^{-}$condensation sets in at a density $2.23 n_{0}$ and the mixed phase ends at $4.1 n_{0}$. On the other hand, $\Lambda$ hyperons appear at a density $2.51 n_{0}$. It is worth mentioning here that we obtained qualitatively similar results with the GM parameter set corresponding to $K=240$ $\mathrm{MeV}$ and $U_{\bar{K}}\left(n_{0}\right)=-140 \mathrm{MeV}$. However, this led to a soft EoS resulting in a neutron star mass below the accurately measured mass (Chatterjee \& Bandyopadhyay 2007b). The composition of $\beta$-equilibrated and charge neutral matter in the presence of $K^{-}$condensate for $U_{\bar{K}}\left(n_{0}\right)=-160 \mathrm{MeV}$ is displayed in Figure 1 . Negatively charged particles such as electrons and muons are depleted from the system with the onset of $K^{-}$condensation and its rapid growth there after. At this stage, the proton density becomes equal to the density of $K^{-}$ mesons in the condensate. It is evident from the figure that $\Lambda$ hyperons populate the system just after the onset of $K^{-}$condensation.

The relaxation time for the non-leptonic process $n+p \rightleftharpoons p+\Lambda$ is plotted with normalised baryon density in Figure 2 for $U_{\bar{K}}\left(n_{0}\right)=-160 \mathrm{MeV}$ and temperature $T=10^{10} \mathrm{~K}$. Here 
equations of state enter as inputs in the calculation of relaxation time in different phases. In particular, partial derivatives of pressure and chemical potentials with respect to neutron number density are calculated using EoS according to the prescription as discussed in section II. This figure shows relaxation time in different phases i.e. the pure antikaon condensed phase (light solid line) and the hadronic (bold solid line) and antikaon condensed parts of the mixed phase (dashed line). We find that the values of relaxation time in the pure and mixed antikaon condensed phases are significantly smaller than that of the hadronic phase involving non-superfluid $\Lambda$ hyperons (Nayyar \& Owen 2006; Chatterjee \& Bandyopadhyay 2006). The relaxation time for the non-leptonic weak process involving hyperons is inversely proportional to $T^{2}$ as given by Eq.(15).

Figure 3 exhibits the hyperon bulk viscosity coefficient as a function of normalised baryon density at a temperature $T=10^{10} \mathrm{~K}$. Similar to Fig. 2, the bulk viscosity in the pure antikaon condensed phase (light solid line) and the hadronic (bold solid line) and antikaon condensed parts of the mixed phase (dashed line) are shown here. One can immediately see that the hyperon bulk viscosity in the antikaon condensed matter, irrespective of whether it is in the pure or mixed phase, is suppressed compared with that of hadronic phase. This suppression may be attributed to the superconducting phase i.e the $K^{-}$condensed phase. The role of superfluidity on hyperon bulk viscosity was studied at length by several groups (Haensel et al. 2002; Nayvar \& Owen 2006; Andersson 2007). They also obtained significant suppression in the hyperon bulk viscosity because one or more superfluid particles were participating in non-leptonic weak processes involving hyperons. In our calculation, $K^{-}$ mesons in the Bose-Einstein condensed state is not a member of the non-leptonic weak process $n+p \rightleftharpoons p+\Lambda$. Therefore, the suppression of hyperon bulk viscosity in our case originates from the EoS in the $K^{-}$condensed phase which enters into the calculation of the chemical imbalance as given by Eq.(17). We further add that the factor $\omega \tau$ in the bulk viscosity coefficient given by Eq.(15) is negligible compared with unity over the whole range of baryon densities considered here. This leads to a $1 / T^{2}$ temperature dependence of the hyperon bulk viscosity. However, the inversion of the temperature dependence was observed in some calculations (Haensel et al. 2002; Nayyar \& Owen 2006) when the factor $\omega \tau$ is much greater than unity. We find a jump in the bulk viscosity coefficient at the upper phase boundary of the mixed phase and pure $K^{-}$condensed phase. This is attributed to kinks in the EoS and discontinuities in $\left(\gamma_{\infty}-\gamma_{0}\right)$.

Now we discuss the results of damping time scale due to the hyperon bulk viscosity and critical angular velocity. This calculation needs the knowledge of the energy density profile, hyperon bulk viscosity profile and the structure of the rotating neutron star in question. For this purpose, we consider a neutron star of gravitational mass $1.60 M_{\odot}$ having baryon rest mass $1.76 M_{\odot}$ and central baryon density $3.50 n_{0}$ and rotating at an angular velocity 
$\Omega_{\text {rot }}=2652 \mathrm{~s}^{-1}$ from the sequence of rotating neutron stars calculated by the model of Stergioulas (Stergioulas \& Friedman 1995). This neutron star contains both $\Lambda$ hyperons and $K^{-}$condensate in its core as its central baryon density is well above the threshold densities of $K^{-}$condensation and $\Lambda$ hyperons. The hyperon bulk viscosity profile of this neutron star as a function of equatorial distance for $T=10^{10} \mathrm{~K}$ is displayed in Figure 4. The hyperon bulk viscosity in the hadronic and antikaon condensed parts of the mixed phase are shown by bold solid and dashed lines, respectively. Further we note that the bulk viscosity profile drops to zero value beyond $3.5 \mathrm{~km}$ because the baryon density beyond this distance decreases below the threshold density of $\Lambda$ hyperons.

As soon as we know the energy density and bulk viscosity profiles, we obtain the damping time scale corresponding to the hyperon bulk viscosity and critical angular velocities as a function of temperature solving $1 / \tau_{r}=0$ for a rotating neutron star mass $1.60 M_{\odot}$. Besides the hyperon bulk viscosity, we also consider the bulk viscosity due to modified Urca process involving nucleons as well as the shear viscosity to the total r-mode time scale as given by Eq.(34). The bulk viscosity due to modified Urca process plays an important role to damp the r-mode at higher temperatures. However, this process can not suppress the r-mode instability below $10^{10} \mathrm{~K}$ because the corresponding damping time scale is longer than the gravitational radiation growth time scale. On the other hand, the shear viscosity coefficient is proportional to $T^{-2}$ and the damping time scale $\tau_{S V}$ is also larger than $\tau_{G R}$ in the temperature range considered here. The bulk viscosity damping time scale $\left(\tau_{B}\right)$ due to the non-leptonic process $n+p \rightleftharpoons p+\Lambda$ and $\tau_{G R}$ are comparable at $\mathrm{T} \sim 4 \times 10^{9}$ and below. Consequently, the $\mathrm{r}$-mode instability is damped in this temperature regime by the hyperon bulk viscosity in $K^{-}$condensed matter. Though the hyperon bulk viscosity is suppressed in the antikaon condensed phase, it is still a very efficient process to damp the r-mode instability.

\section{Summary and Conclusions}

We have investigated the hyperon bulk viscosity due to the non-leptonic weak process $n+p \rightleftharpoons p+\Lambda$ in a $K^{-}$condensed phase and later applied it to study the r-mode instability in neutron stars. For the parameter set adopted here and antikaon optical potential depth $U_{\bar{K}}\left(n_{0}\right)=-160 \mathrm{MeV}, K^{-}$condensation occurs before $\Lambda$ hyperons are populated in the system. We find that the hyperon bulk viscosity coefficient in $K^{-}$condensed matter is significantly suppressed compared with the non-superfluid hyperon bulk viscosity coefficient in the hadronic phase. Further we note that the hyperon bulk viscosity in the superconducting phase is still an efficient process to damp the r-mode instability. 
DB thanks the Alexander von Humboldt Foundation for the support. He also thanks Horst Stöcker and Walter Greiner for their support and acknowledges the warm hospitality at the Institute for Theoretical Physics, J.W. Goethe University, Frankfurt am Main and Frankfurt Institute for Advanced Studies (FIAS) where a part of this work was completed.

\section{REFERENCES}

Alford, M. G., \& Schmitt, A. 2007a, J. Phys. G, 34, 67

Alford, M. G., Braby, M., Reddy, S., \& Schafer, T. 2007b, Phys. Rev. C, 75, 055209

Alford, M. G., Schmitt, A., Rajagopal, K., \& Schafer, T. 2007c, preprint (arXiv:0709.4635)

Andersson, N. 1998, ApJ, 502, 708

Andersson, N., Kokkotas, K. D., \& Schutz, B. F. 1999, ApJ, 510, 846

Andersson, N., \& Kokkotas, K. D. 2001, Int. J. Mod. Phys., D10, 381

Andersson, N. 2003, Class. Quant. Grav., 20, R105

Andersson, N. 2007, Astrophys. Space Sci. 308, 395

Banik, S., \& Bandyopadhyay, D. 2001a, Phys. Rev. C, 63, 035802

Banik, S., \& Bandyopadhyay, D. 2001b, Phys. Rev. C, 64, 055805

Batty, C. J., Friedman, E., \& Gal, A. 1997, Phys. Rep., 287, 385

Boguta, J., \& Bodmer, A. R. 1977, Nucl. Phys., A292, 413

Chakrabarty, D. et al., 2003, Nature, 424, 42

Chakrabarty, D. 2005, in ASP Conference Series 328, Binary Radio Pulsars, ed. F. Rasio, \& I. Stairs (Aspen,Colorado:ASP), 279

Chandrasekhar, S. 1970, Phys. Rev. Lett., 24, 611

Chatterjee, D., \& Bandyopadhyay, D. 2006, Phys. Rev. D 74, 023003

Chatterjee, D., \& Bandyopadhyay, D. 2007a, Ap\&SS, 308, 451

Chatterjee, D., \& Bandyopadhyay, D. 2007b, Phys. Rev. D 75, 123006 
Chrien, R. E., \& Dover, C. B. 1989, Annu. Rev. Nucl. Part. Sci., 39, 113

Dalen, van E. N. E., \& Dieperink, A. E. L. 2004, Phys. Rev. C, 69, 025802

Drago, A., Lavagno, A., \& Pagliara, G. 2005, Phys. Rev. D, 71, 103004

Dong, H., Su, N., \& Wang, Q. 2007a, Phys. Rev. D, 75, 074016

Dong, H., Su, N., \& Wang, Q. 2007b, J. Phys. G, 34, 6643

Dover C. B., \& Gal, A. 1984, Prog. Part. Nucl. Phys. 12, 171

Friedman, E., Gal, A., \& Batty, C. J. 1994, Nucl. Phys. A, 579, 518

Friedman, E., Gal, A., Mareš, J., \& Cieplý, A. 1999, Phys. Rev. C, 60, 024314

Friedman, J. L. 1978, Commun. Math. Phys., 62, 247

Friedman, J. L., \& Schutz, B. F. 1978a, ApJ, 221, 937

Friedman, J. L., \& Schutz, B. F. 1978b, ApJ, 222, 281

Friedman, J. L., \& Morsink, S. M. 1998, ApJ, 502, 714

Glendenning, N. K. 1992, Phys. Rev. D, 46, 1274

Glendenning, N. K., \& Schaffner-Bielich, J. 1998, Phys. Rev. Lett., 81, 4564

Glendenning, N. K., \& Schaffner-Bielich, J. 1999, Phys. Rev. C, 60, 025803

Glendenning, N. K., \& Moszkowski, S. A. 1991, Phys. Rev. Lett., 67, 2414

Haensel, P., Levenfish, K. P., \& Yakovlev, D. G. 2000, A\&A, 357, 1157

Haensel, P., Levenfish, K. P., \& Yakovlev, D. G. 2001, A\&A, 372, 130

Haensel, P., Levenfish, K. P., \& Yakovlev, D. G. 2002, A\&A, 381, 1080

Hessels, J. W. T. et al., 2006, Science, 311, 1901

Jones, P. B. 2001a, Phys. Rev. Lett., 86, 1384

Jones, P. B. 2001b, Phys. Rev. D, 64, 084003

Koch, V. 1994, Phys. Lett. B, 337, 7

Landau, L. D., \& Lifshitz, E. M. 1999, Fluid Mechanics (Oxford:Butterworth-Heinemann) 
Li, G. Q., Lee, C.-H., \& Brown, G. E. 1997a, Phys. Rev. Lett., 79, 5214

Li, G. Q., Lee, C.-H., \& Brown, G. E. 1997b, Nucl. Phys. A, 625, 372

Lindblom, L., Owen, B. J., \& Morsink, S. M. 1998, Phys. Rev. Lett., 80, 4843

Lindblom, L., Mendell, G., \& Owen, B. J. 1999, Phys. Rev. D, 60, 064006

Lindblom, L., \& Mendell, G. 2000, Phys. Rev. D, 61, 104003

Lindblom, L., \& Owen, B. J. 2002, Phys. Rev. D, 65, 063006

Madsen, J. 1992, Phys. Rev. D, 46, 3290

Madsen, J. 2000, Phys. Rev. Lett., 85, 10

Nayyar, M. \& Owen, B. J. 2006, Phys. Rev. D 73, 084001

Pal, S., Ko, C. M., Lin, Z., \& Zhang, B. 2000, Phy. Rev. C, 62, 061903

Pan, N. N., Zheng, X. P., \& Li, J. R. 2006, MNRAS, 371, 1359

Sa'd, B. A., Shovkovy, I. A., \& Rischke, D. H. 2007a, Phys. Rev. D, 75, 065016

Sa'd, B. A., Shovkovy, I. A., \& Rischke, D. H. 2007b, Phys. Rev. D, 75, 125004

Sawyer, R. F. 1989, Phys. Rev. D, 39, 3804

Schaffner, J., Dover, C. B., Gal, A., Greiner, C., \& Stöcker, H. 1993, Phys. Rev. Lett., 71, 1328

Schaffner, J., Dover, C. B., Gal, A., Millener, D. J., Greiner, C., \& Stöcker, H. 1994, Ann. Phys. (N.Y.), 235, 35

Schaffner, J., \& Mishustin, I. N. 1996, Phys. Rev. C, 53, 1416

Serot, B. D., \& Walecka, J. D. 1986, Adv. in Nucl. Phys., 16, 1

Stergioulas, N. 2003, Liv. Rev. Rel., 6, 3

Stergioulas, N., \& Friedman, J. L. 1995, ApJ, 444, 306

Waas, T., \& Weise, W. 1997, Nucl. Phys. A, 625, 287 


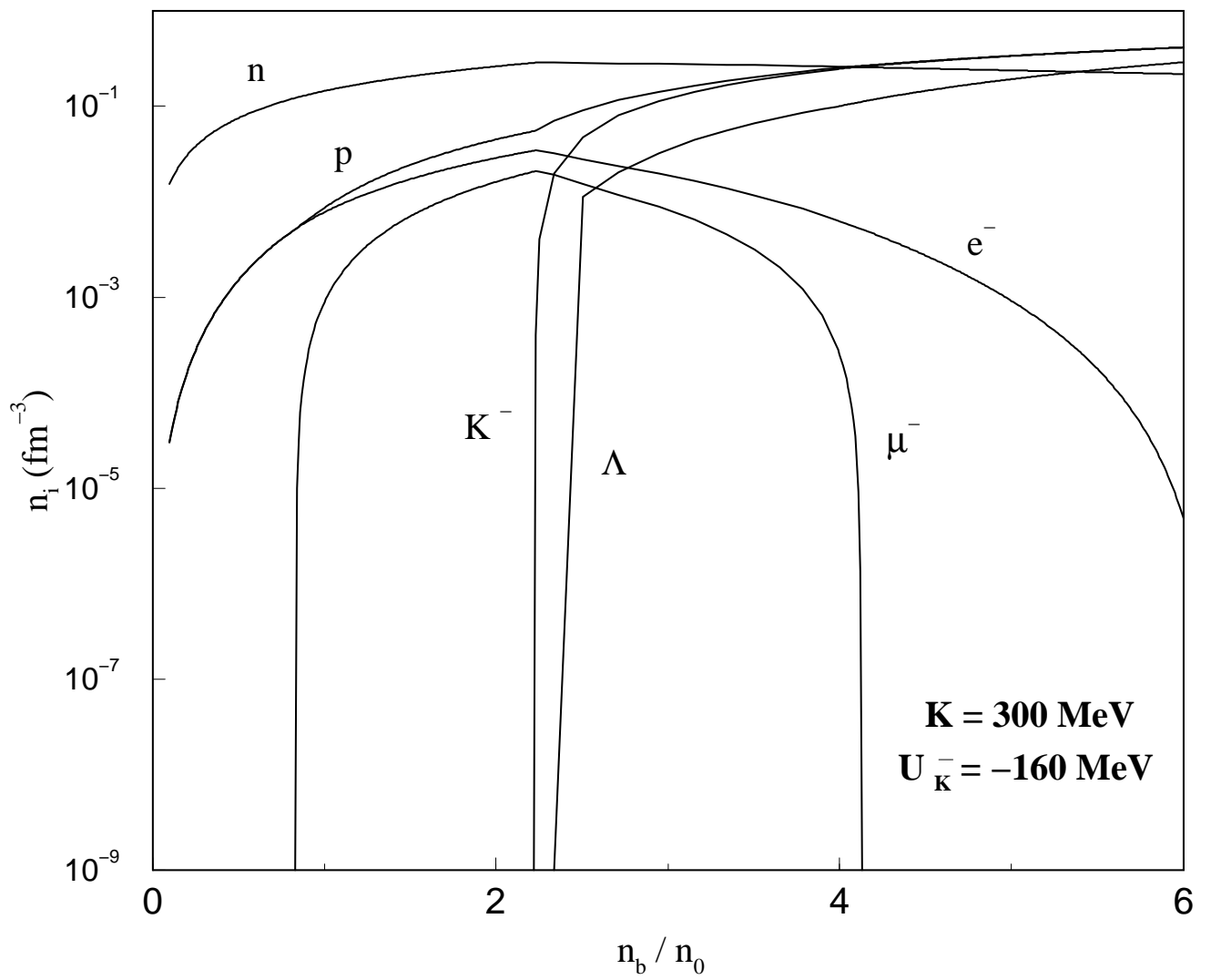

Fig. 1.- Particles abundances are plotted with normalised baryon density for antikaon optical potential depth at normal nuclear matter density $U_{\bar{K}}\left(n_{0}\right)=-160 \mathrm{MeV}$. 


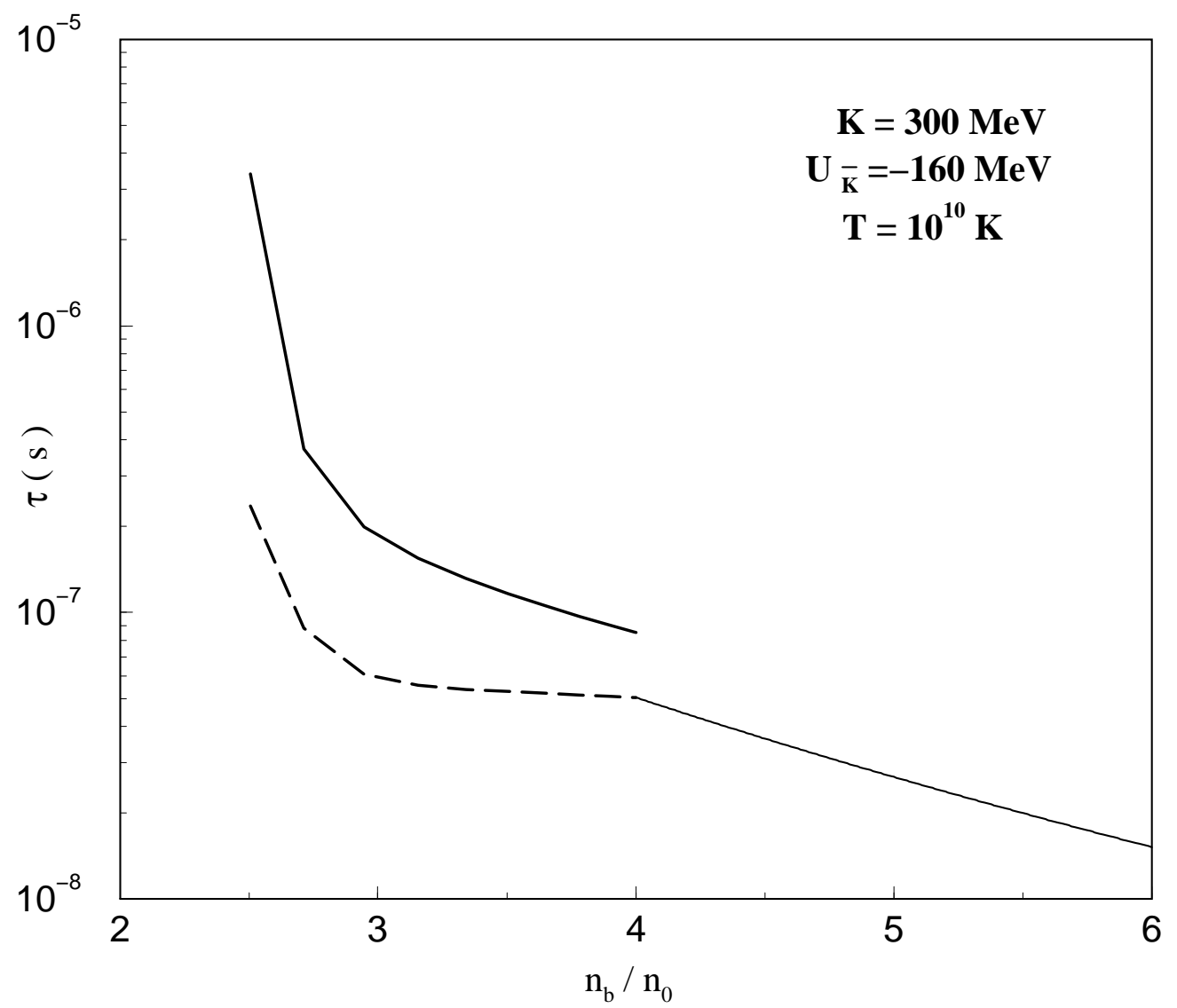

Fig. 2.- Relaxation time is plotted with normalised baryon density for the non-leptonic process $n+p \rightleftharpoons p+\Lambda$ and antikaon optical potential depth at normal nuclear matter density $U_{\bar{K}}\left(n_{0}\right)=-160 \mathrm{MeV}$. The contributions of the pure antikaon condensed phase and the hadronic and antikaon condensed parts of the mixed phase are shown by light solid, bold solid and dashed lines, respectively. 


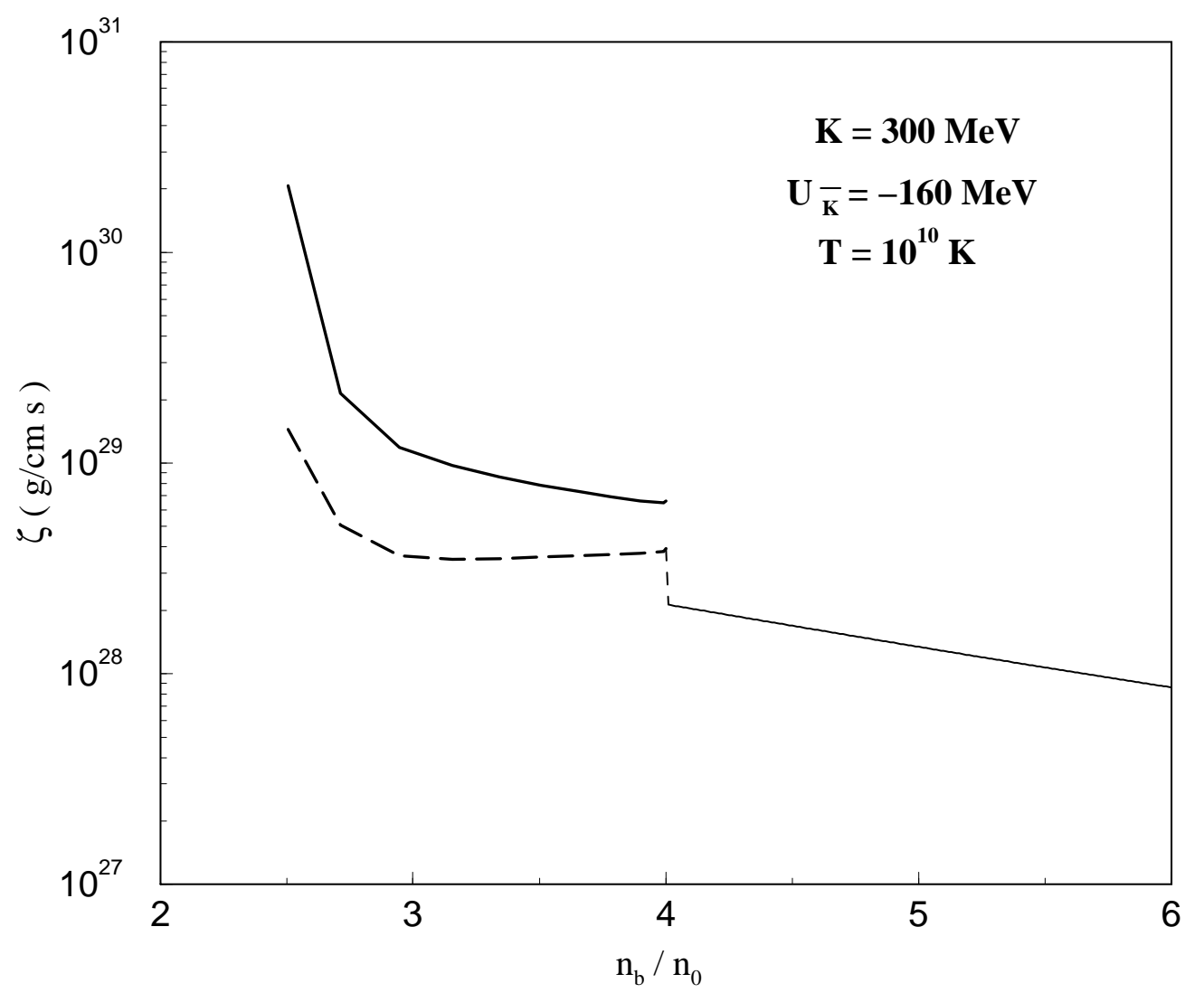

Fig. 3.- Hyperon bulk viscosity coefficient is exhibited as a function of normalised baryon density at a temperature $10^{10} \mathrm{~K}$ and antikaon optical potential depth at normal nuclear matter density $U_{\bar{K}}\left(n_{0}\right)=-160 \mathrm{MeV}$. Different lines have the same meaning as in Fig. 2. 


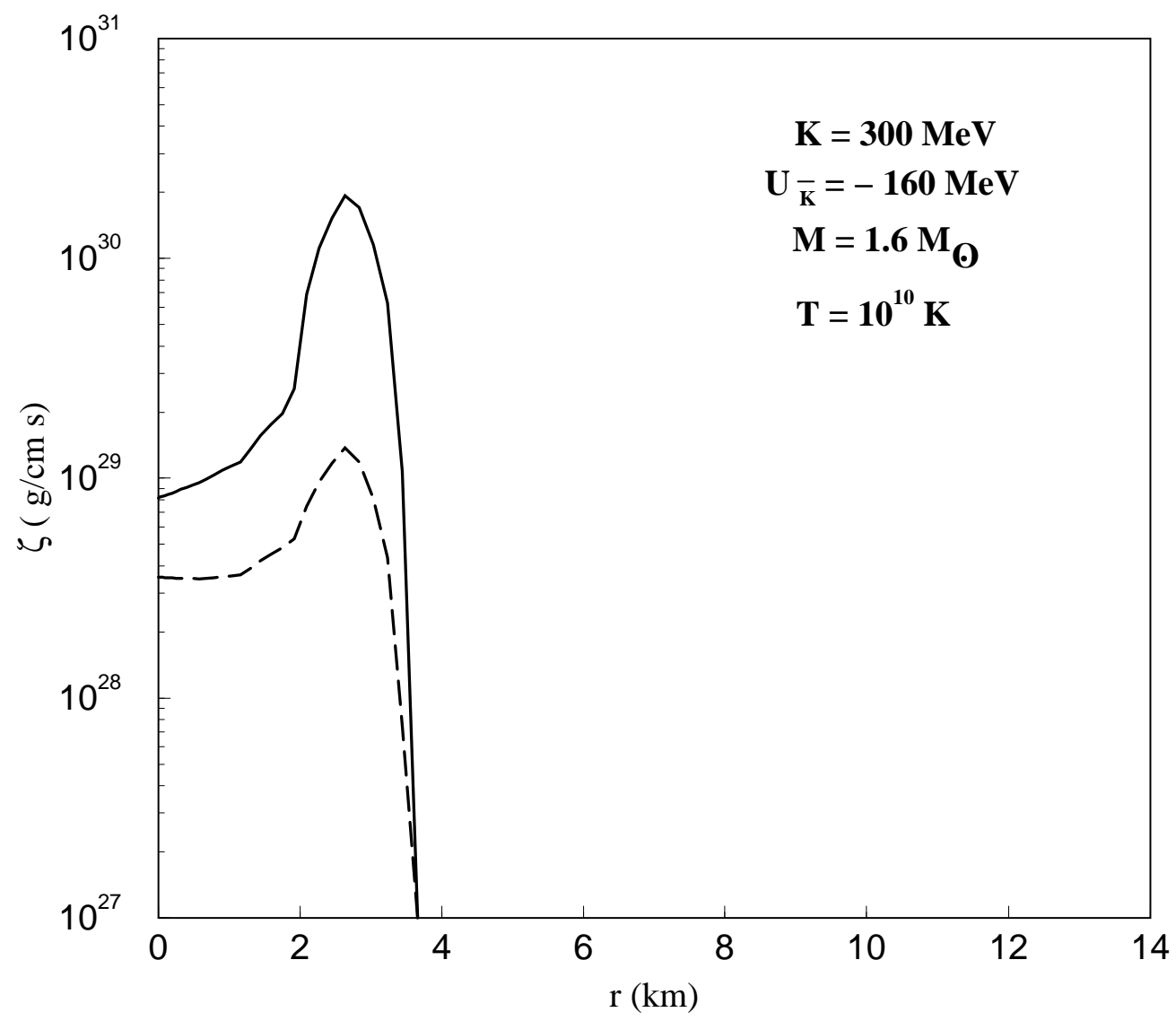

Fig. 4.- Hyperon bulk viscosity profile is shown with equatorial distance for a rotating neutron star of mass $1.60 \mathrm{M}_{\odot}$ at a temperature $10^{10} \mathrm{~K}$ and antikaon optical potential depth $U_{\bar{K}}\left(n_{0}\right)=-160 \mathrm{MeV}$. The hadronic and antikaon condensed parts of the mixed phase are shown by bold solid and dashed lines, respectively. 


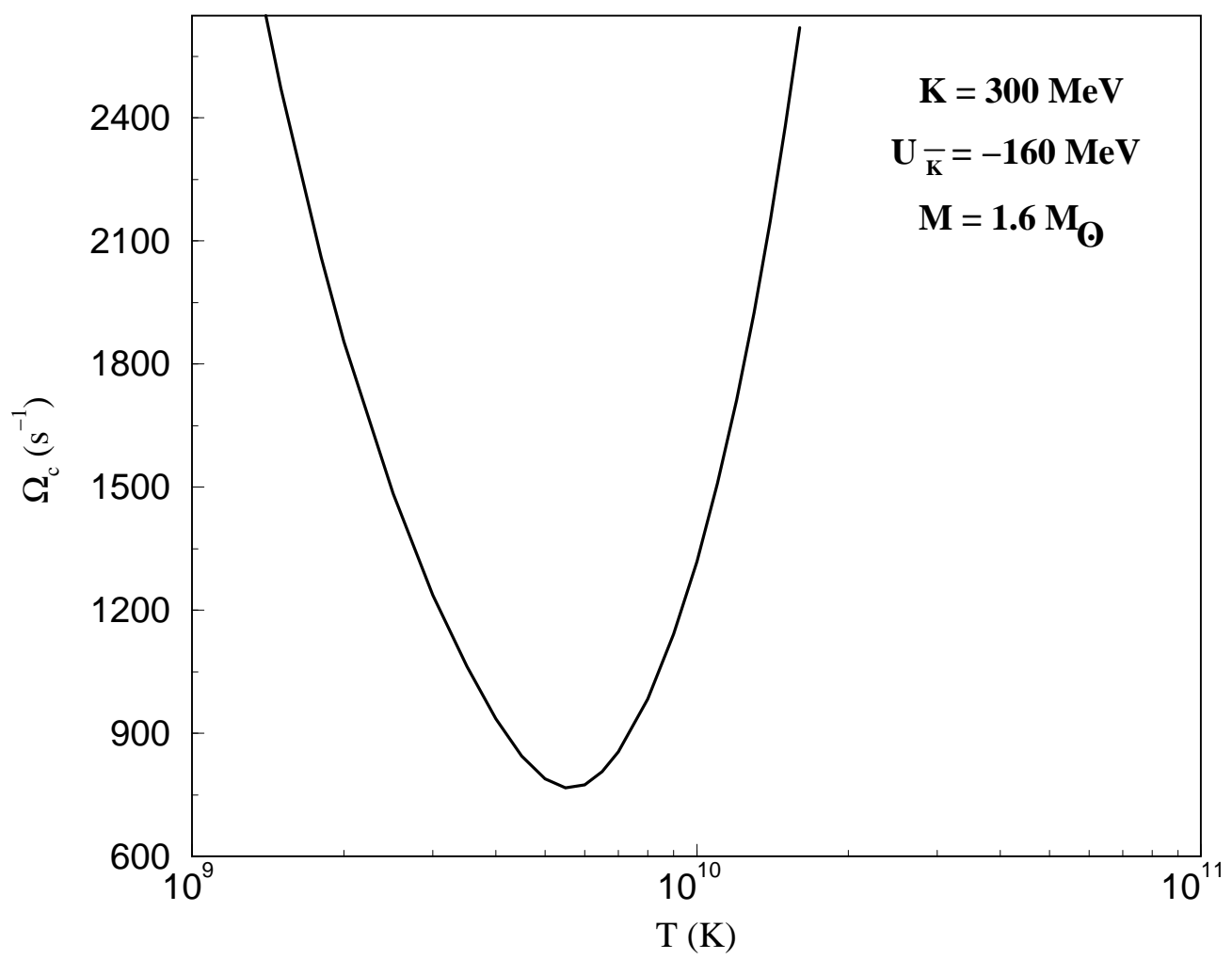

Fig. 5.- Critical angular velocity for $1.60 \mathrm{M}_{\odot}$ neutron star is plotted as a function of temperature. 